\title{
Experimental Study on the Combined Killing Effect of DC and CIK Cells on Gastric Cancer Stem Cells
}

\author{
Zhang Lin ${ }^{1}$, Hou Yanhong ${ }^{1,}$, Zhang Jian ${ }^{2}$, Wang Yanmei ${ }^{1}$ \\ ${ }^{1}$ Department of Gastroenterology, The Eighth Medical Center of PLA General Hospital, Beijing, China \\ ${ }^{2}$ Department of molecular biology, Air Force Military Medical University of PLA, Xi'an, China
}

Email address:

stepinghuns2@aliyun.com (Hou Yanhong)

${ }^{*}$ Corresponding author

\section{To cite this article:}

Zhang Lin, Hou Yanhong, Zhang Jian, Wang Yanmei. Experimental Study on the Combined Killing Effect of DC and CIK Cells on Gastric Cancer Stem Cells. Biomedical Sciences. Vol. 7, No. 1, 2021, pp. 20-24. doi: 10.11648/j.bs.20210701.14

Received: January 23, 2021; Accepted: February 8, 2021; Published: February 23, 2021

\begin{abstract}
Background: Gastric cancer is one of the most fatal malignant tumors in China. Gastric cancer stem cells are the key factors of tumor recurrence and metastasis. In recent years, immunotherapy for gastric cancer stem cells has become a research hotspot. Objective: to preliminarily verify the combined killing effect of DC and CIK cells on gastric cancer stem cells. To evaluate the effect of DC-CIK cells as a potential therapeutic agents for gastric cancer. Methods: Based on human gastric cancer cell line SGC7901, gastric cancer stem cells were isolated and cultured by immunomagnetic beads. The cytotoxicity of DC-CIK effector cells to gastric cancer stem cells was investigated by ${ }^{51} \mathrm{Cr}$ killing test. The cell cycle and apoptosis of tumor stem cells were detected by flow cytometry. Then the comparisions of the cytotoxicity activity between DC-CIK and CIK only was conducted. Results: the gastric cancer stem cells isolated and cultured by CD44 and CD133 immunomagnetic beads sorting method met the research requirements. ${ }^{51} \mathrm{Cr}$ cell killing test results showed that the killing rate of DC-CIK combined as effector cells on gastric cancer SGC7901 stem cell line was significantly higher than those of the control group $(\mathrm{P}<0.05)$. After DC-CIK effector cells treatment, the apoptosis rate of tumor stem cells increased significantly. Conclusion: preliminary in vitro experiments show that DC-CIK cells combined as effector cells have obvious killing effect on gastric cancer stem cells, which could has potential clinical application value for controlling recurrence and metastasis of gastric cancer after surgery, radiotherapy and chemotherapy.
\end{abstract}

Keywords: Gastric Cancer, Cellular Immunotherapy, DC Cells, CIK Cells, Stem Cells

\section{Background}

Gastric cancer is the most common malignant tumor in China. The rise of incidence rate or mortality rate in recent years are slightly slower than other malignant tumors, but gastric cancer still remain at the forefront of overall incidence rate. It is a major disease that seriously endangering the life and health of Chinese people. With the popularization of endoscopy in China, the early diagnosis rate of gastric cancer has been greatly improved. Many patients have received surgery, chemotherapy, radiotherapy and other adjuvant treatment earlier, but tumor recurrence and metastasis still seriously threaten the lives of patients. With the rapid development of cell biology in recent years, researchers have realized that cancer stem cells are the source of recurrence and metastasis of malignant tumors. The existence of gastric cancer stem cells and its biological significance in tumor recurrence and metastasis have also been confirmed [1-9]. In recent years, with the continuous progress of tumor treatment technology, the emergence and large-scale application of cell biological therapy technology has become a new hope for human tumor treatment. At present, NK cells, CTL cells, CIK cells and DC-CIK cells have been used in clinical treatment of gastric cancer. Early clinical observation has a certain therapeutic effect on such patients. Our previous animal experimental studies also suggested that CIK cells as the main biological cell immunotherapy has a good killing effect on gastric cancer cells [10]. However, studies on the killing effect of immune effector cell therapy on gastric cancer stem cells are rare. The purpose of this study was to verify the killing ability of DC-CIK cells on gastric cancer stem cells in vitro. 


\section{Materials and Methods}

\subsection{Materials}

Gastric cancer cell lines SGC7901, DC cells, CIK cells, etc. were stored in the laboratory of 309 Hospital of Chinese PLA. Ordinary cell culture plates and culture bottles were purchased from orange company of Belgium, inverted phase contrast microscope and fluorescence microscope were purchased from Olympus company of Japan. Anti-CD44 antibody and anti-CD133 antibody were purchased from Abcam company.

\subsection{Methods}

\subsubsection{The Preparation of Gastric Cancer Stem Cells}

Gastric cancer stem cells were prepared by flow cytometry immunosorting method according to Huang Lei et al [11]. SGC7901 cells were cultured under conventional conditions at $37^{\circ} \mathrm{C}$ and $5 \% \mathrm{CO}_{2}$, and cultured in complete medium to logarithmic growth phase. The cells in logarithmic growth phase were washed with PBS, digested with trypsin, resuspended with PBS and counted. The cells were mixed with FITC-CD44 antibody and PE-CD133 antibody (final concentration $5 \mu \mathrm{g} / \mathrm{ml}$ ), and incubated at $37^{\circ} \mathrm{C}$ for $30 \mathrm{~min}$. After washing with PBS buffer for 3 times, the cells were selected by flow cytometry. Then the selected cells were resuspended in complete culture medium, and they were observed under inverted microscope after cells adhered to the wall. The observation results were as shown in Figure 1, and then continued to culture for standby.

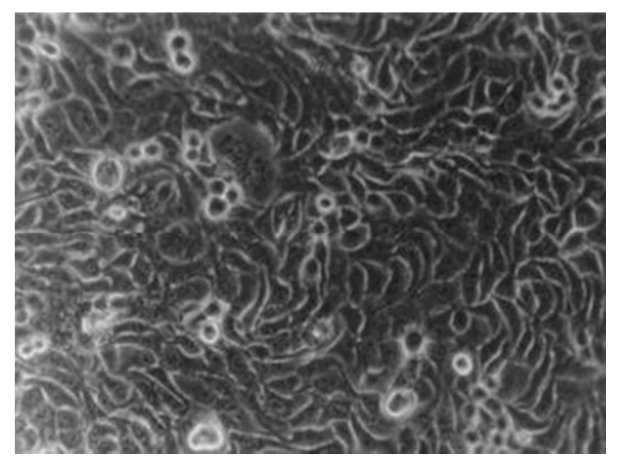

Figure 1. The results of sorting gastric cancer stem cells under inverted microscope. The cells were slightly spindle and polygonal, and arranged closely.

\subsubsection{The Culture of DC-CIK Cells}

DC-CIK cells were cultured in combination with the previous methods of our research group [10] and Zhao Xin et al [12], peripheral blood mononuclear cells (PBMC) from healthy adults were isolated and collected by COBE spectra blood cell separator. PBMCs were extracted by Ficoll density gradient centrifugation. The PBMCs were washed with RPMI-1640 medium for 3 times. The cells were suspended in RPMI-1640 complete medium with INF $-\gamma 500 \mathrm{U} / \mathrm{ml}$ for $24 \mathrm{~h}$ under the condition of $37^{\circ} \mathrm{C}$ and $5 \% \mathrm{CO}_{2}$. Then, IL-2 $400 \mathrm{U} / \mathrm{ml}$, IL-1 $\alpha 100 \mathrm{U} / \mathrm{ml}$ and human CD3 McAb $10 \mathrm{ng} / \mathrm{ml}$ were added and they were continuously cultured. the growth of cells was observed regularly. The medium was changed every 2 days and the number of cells was adjusted to $2 \times 10^{6} / \mathrm{ml}$. after 10 days of continuous culture, the viable cells were counted by trypan blue. After 15-24 days of cell culture, a few cultured cells were detected by flow cytometry. When the number of CD3 and CD56 double positive cells were more than 50\%, CD3 and CD8 double positive cells were more than $30 \%$, these effector cells could be used in the experiment. DC cells were stimulated to mature by freeze-thaw antigen of human SGC7901 gastric cancer cells prepared by repeated freeze-thaw method. DC cells and CIK cells cultured for 7 days were mixed with CIK cells at a ratio of 1:5. IL-2 $(300 \mathrm{u} / \mathrm{ml})$ was added every 3 days for one week. The killing test was carried out and the observation results under inverted microscope were shown in Figure 2.

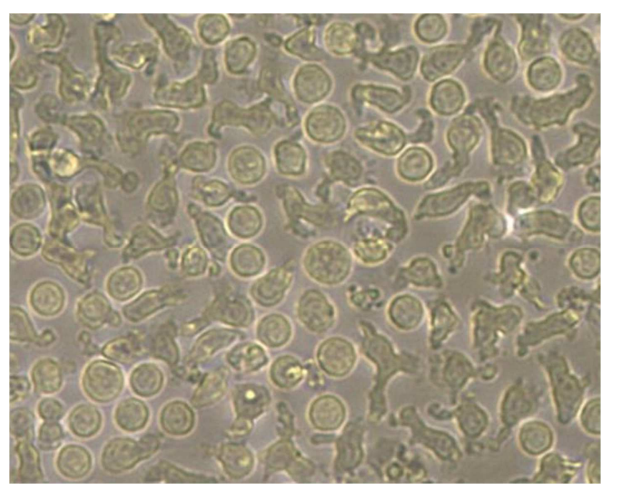

Figure 2. The mixed culture of DC and CIK cells was observed under inverted microscope. The results showed that the DC cells were slightly irregular with buds and CIK cells were round.

\subsubsection{The Killing Rate of DC-CIK Effector Cells}

The killing rate of DC-CIK effector cells on gastric cancer stem cells was detected by ${ }^{51} \mathrm{Cr}$ cell killing detection method [13]. SGC7901 gastric cancer stem cells in logarithmic growth phase were inoculated with $1 \times 10^{6}$ in 6 well plate, incubated overnight with $300 \mathrm{u}{ }^{51} \mathrm{Cr}$, washed three times, and the cell concentration was adjusted to $2 \times 10^{4} / \mathrm{ml}$. $100 \mu \mathrm{L}$ target cells labeled with ${ }^{51} \mathrm{Cr}$ were inoculated into 96 well plate. The ratio of effector cells (counting CIK cells) to target cells was set at 75: 1 and the total volume was $200 \mu \mathrm{L}$. After mixing, the target cells were incubated with $5 \% \mathrm{CO}_{2}$ at $37^{\circ} \mathrm{C}$ for $6 \mathrm{~h}$, and $100 \mu \mathrm{L}$ supernatant was centrifuged and measured by gamma scintillation counter. At the same time, natural release pore (target cells plus culture medium group) and maximum release pore (target cells plus 1\% Triton group) were set up. Each experimental group was added with different components according to the following, and each group was set with 5 repeated controls. Group A: pure DC cells $\left(0.3 \times 10^{6} / \mathrm{ml}\right)+$ gastric cancer stem cells $\left(2 \times 10^{4} / \mathrm{ml}\right)$; group B: simple CIK cells $\left(1.5 \times 10^{6} / \mathrm{ml}\right)+$ gastric cancer stem cells $\left(2 \times 10^{4}\right.$ $/ \mathrm{ml})$; group C: DC-CIK cells $\left(0.3 \times 10^{6} / \mathrm{ml}, 1.5 \times 10^{6} / \mathrm{ml}\right)+$ gastric cancer stem cells $\left(2 \times 10^{4} / \mathrm{ml}\right)$; roup D: PBS buffer solution+gastric cancer stem cells $\left(2 \times 10^{4} / \mathrm{ml}\right)$ (blank control). The killing rate was calculated according to the following formula.

Kill rate $=(C M P$ value of experimental pore-CMP value of natural release pore) /(CMP value of maximum release-CMP value of natural release pore) $\times 100 \%$ 


\subsubsection{Cell Cycle and Apoptosis Were Detected by Flow Cytometry}

According to our previous study [10], the gastric cancer stem cells in experimental groups were isolated after 24 hours of coculture in complete medium. The serum-free medium was used for 24 hours, and then the complete medium was used for 24 hours. After pancreatin digestion, the cells were washed with PBS ( $\mathrm{pH}=8.0)$, centrifuged and stained with propidium iodide (PI) for $10 \mathrm{~min}$. Then the cell cycle was detected by flow cytometry. The experiment was repeated 5 times, and the average value was compared. The cells in each group were synchronized and centrifuged according to the above methods, and then stained with anexin V-FITC for $30 \mathrm{~min}$, PI staining for $10 \mathrm{~min}$, and the apoptosis was detected by flow cytometry.

\subsubsection{Transmission Electron Microscopic Observation of Gastric Cancer Stem Cells after Treatment}

After treatment, the stem cells were resuspended with PBS, washed three times, centrifuged again as cell mass, fixed with glutaraldehyde solution, embedded, and sectioned to observe the microstructural changes by transmission electron microscope.

\subsection{Statistical Treatment}

SPSS11.0 software was used for statistical analysis, and two factor and single factor analysis of variance were used for statistical analysis.

\section{Results}

\subsection{The Results of ${ }^{51} \mathrm{Cr}$ Cell Killing Detection}

The killing rate of DC-CIK cells in group C was $45.1 \pm 6.2 \%$, which was significantly higher than that of other control groups $(\mathrm{P}<0.05)$, and that of group $\mathrm{B}$ was significantly higher than that of the other two groups $(\mathrm{P}<0.05)$. The specific results are shown in Figure 3.

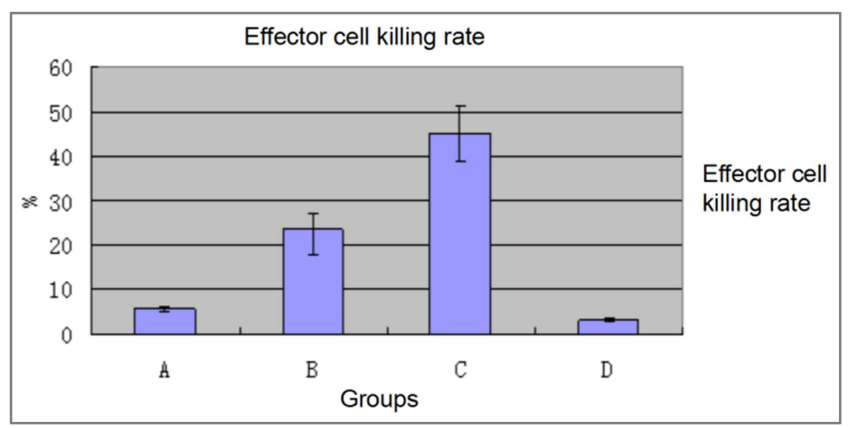

Figure 3. It can be seen that group B and group C have definite killing effect, while group A and group d have no obvious killing effect, among which group $C$ is significantly higher than group $B$.

\subsection{The Results of Cell Cycle Detection by Flow Cytometry}

The results of the cell cycle of gastric cancer stem cells in each group after treatment showed that there was no significant difference in the cell cycle of each group after treatment. The specific results are shown in Table 1.

\subsection{The Results of Apoptosis}

The results of apoptosis were detected by flow cytometry The apoptosis rate of gastric cancer stem cells in group $\mathrm{C}$ was $6.4 \%$, which was significantly higher than that in group $\mathrm{A}$ $(3.1 \%), 2.6 \%$ and $3.4 \%$ in group B and D.

Table 1. There was no significant difference in the cell cycle of each group in Table 1, and the effect of effector cell therapy on the cycle change of cancer stem cells was not obvious.

\begin{tabular}{lllll}
\hline Group & n & G0-G1 (\%) & G2-M (\%) & S (\%) \\
\hline A & 5 & $50.13 \pm 4.55$ & $12.66 \pm 5.17$ & $37.21 \pm 7.07$ \\
B & 5 & $48.66 \pm 4.76$ & $14.45 \pm 5.21$ \\
C & 5 & $52.32 \pm 7.06$ & $14.79 \pm 5.61$ \\
D & 5 & $48.98 \pm 7.65$ & $16.04 \pm 3.77$ \\
\hline
\end{tabular}

\subsection{The Results After Treatment}

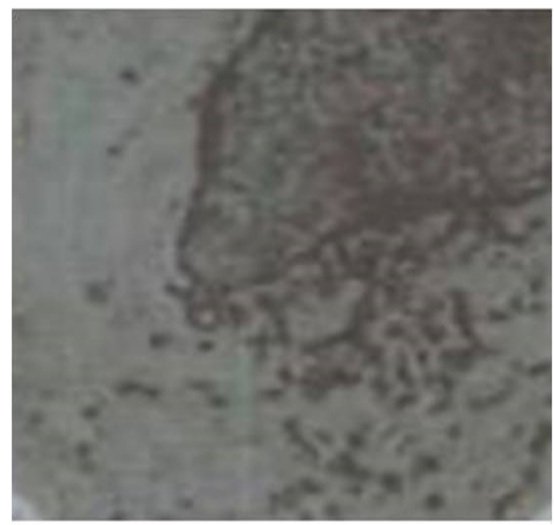

Figure 4. It shows the microstructure changes of group C cells after treatment, endoplasmic reticulum expansion, and widespread distribution of high electron density fine particles in the cells.
After treatment, the gastric cancer stem cells in group B and group $\mathrm{C}$ showed obvious cell necrosis, normal cell structure damage, such as changes in cell morphology, volume reduction, endoplasmic reticulum expansion, and widespread distribution of high electron density fine particles in the cells. In group A and group D, there was no obvious cell necrosis and ultrastructural changes. (Figure 4)

\section{Conclusion}

Gastric cancer stem cells are considered to be closely related to tumor recurrence and metastasis. In this study, we examined the killing effect of DC cells combined with CIK cells on gastric cancer stem cells. According to the experimental results, DC cells combined with CIK cells have certain killing ability on gastric cancer stem cells, which can effectively inhibit the proliferation of cells and promote their apoptosis. 


\section{Discussion}

The incidence rate of gastric cancer has long been the highest in the digestive tract cancer. Most of the patients with gastric cancer in China are in the advanced stage. The treatment of surgery and traditional treatment is poor. At present, it is considered that the existence of cancer stem cells is an important cause of recurrence and metastasis. However, the killing effect of conventional radiotherapy and chemotherapy on these cells is limited. Biological immunotherapy has been applied in clinical practice, and has become another powerful weapon in medical treatment of tumor after traditional chemotherapy and radiotherapy. In our previous research [10], similar methods were used to detect the killing effect of CIK cells on gastric cancer cells, and some experimental results were obtained. DC-CIK cells have been used to treat solid tumors in vitro, in animals and in clinical humans. In domestic studies, Mu Ying et al [14] used DC-CIK cells to kill human colon cancer HT29 in vitro. The results indicate that such effector cells have strong killing effect on colon cancer cells. Zheng Bing et al [15] used DC-CIK cells to kill HepG2 cells in vitro, and the research results also showed that this method has a good killing effect on tumor cells. Pang Chong et al [16] used DC-CIK cells derived from tumor stem cells to kill renal cancer cell line A498 and lung cancer cell line A549. The results showed that DC-CIK cells derived from tumor stem cells could kill their homologous tumor cells more effectively. However, in general, this kind of research is rare. So far, there is no search for such research reports on gastric cancer stem cells. Our results show that DC-CIK cells have a high killing effect on gastric cancer stem cells in vitro. The apoptosis of gastric cancer stem cells after treatment has significant changes, and the ultrastructure of DC-CIK cells also has some changes under the electron microscope. Some results suggest that DC-CIK cell immunotherapy may have a certain clinical application prospect in controlling the recurrence and metastasis of gastric cancer.

There are also some problems in this study. First of all, we conducted cytological experiments in vitro. At present, it is difficult to explain the killing and therapeutic effects of DC-CIK cells on gastric cancer stem cells in vivo. Therefore, in the next research, we plan to carry out the killing research on gastric cancer stem cells under the conditions of animal model in vivo. In addition, the research methods in this study are relatively rough. In the next step, we will continue to study with more detailed research methods. At the same time, with the development of molecular targeted drugs [17, 18], as a method to cooperate with immunotherapy, it can enhance and complement each other. Molecular targeted drugs will also be introduced in the research to provide new treatment options for patients with advanced gastric cancer.

\section{References}

[1] Naohide Oue, Kazuhiro Sentani, Naoya Sakamoto, et al. Molecular carcinogenesis of gastric cancer: Lauren classification, mucin phenotype expression, and cancer stem cells [J]. International Journal of Clinical Oncology, 2019, 24: 771-778.
[2] Lingfeng $\mathrm{Fu}$, Luke Bu, Tadahito Yasuda, et al. Gastric Cancer Stem Cells: Current Insights into the Immune Microenvironment and Therapeutic Targets [J]. Biomedicines, 2020, 8 (1): 120-130.

[3] Yue Zhao, Fei Feng, Yong-Ning Zhou, et al. Stem cells in gastric cancer [J]. World Journal of Gastroenterology, 2015, 1: 112-123.

[4] Zengfu Xue, Hui Yan, Juntang Li, et al. Identification of cancer stem cells in vincristine preconditioned SGC7901 gastric cancer cell line [J]. Journal of cellular biochemistry, 2012, 113 (1): 302-312.

[5] Moo-In Park, Jeonghoon Heo, et al. Role of Gastric Stem Cells in Gastric Carcinogenesis by Chronic Helicobacter pylori Infection [J]. The Korean Journal of Helicobacter and Upper Gastrointestinal Research, 2015, 15 (3): 155-159.

[6] Masakazu Yashiro. Gastric Cancer Stem Cells and Resistance to Cancer Therapy [J]. Chemotherapy, 2014, 3: 3.

[7] Ji Hongmin, Bu Peng, Li Feng. Progress of gastric cancer stem cells [J]. Cancer Research and Clinic, 2018, 30 (2): 135-138.

[8] Cheng Jianxin, Wang Junhui, Peng Yonghai, et al. Research on gastric cancer stem cells: current status, problems and future directions [J]. Chinese Journal of Oncology, 2014, 36 (11): 801-804.

[9] MENG Xueping, WANG Yuping, GUO Qinghong, et al. Research progress of gastric cancer stem cells $[\mathrm{J}]$. Chinese Journal of Gastroenterology and Hepatology, 2015, 24 (10): 1166-1170.

[10] ZHANG Lin, HOU Yanhong, DUAN Yunhui, et al. The cytotoxicity activity of CIK cells against gastric cancer cell lines mediated by bispecific antibody [J]. CHINESE JOURNAL OF GASTROENTEROLOGY AND HEPATOLOGY, 2010, 19 (11): 978-981.

[11] HUANG Lei, HUANG Xia, CAI Yiting, et al. Study on the effect of HSP90 inhibitor IPI-504 on gastric cancer cells and its stem cell like cells [J]. Modern Journal of Integrated Traditional Chinese and Western Medicine. 2015, 24 (30): 3317-3320.

[12] Zhao Xin, Chang Ying, Liu Yuxia, et al. Experiment study of killing effect of DC and CIK co-cultured on tumor cells [J]. Chinese Journal of Laboratory Diagnosis, 2015, 19 (10): 1636-1638.

[13] Yang Wenlan, Cui Heng, Feng Jie, et al. In vitro study on cytotoxicity of $\mathrm{T}$ cells induced by dendritic cells loaded with 6B11 anti-idiotypeminibody to ovarian cancer cell lines $[\mathrm{J}]$. CHINESE JOURNAL OF CLINICAL OBSTETRICS AND GYNECOLOGY. 2004, 5 (2): 127-131.

[14] MU Ying, WANG Weihua, CHEN Shuangfeng, et al. Comparison of killing effects of human peripheral blood and umbilical cord blood-derived DC-CIK on colon cancer HT29 cells [J]. Shandong Medical Journal, 2016, 56 (40): 13-16.

[15] ZHENG Bing, XIE Fang-Yi, CAI Guo-Hui, et al. Effects of Ophiocordyceps xuefengensis on proliferation of DC-CIK cells and activity of killing HepG-2 cells by DC-CIK cells [J]. Chinese Journal of Immunology, 2015, (2): 189-192.

[16] PANG Chong, ZHANG Tengyue, WANG Changli, et al. Effect of Tumor Stem Cell Derived CSC-DC-CIK on Destructing Homologous Tumor Cells [J]. Tianjin Medical Journal, 2014, 42 (10): 973-976. 
[17] Tingting Pan, Weizuo Chen. Advances in targeted therapy of gastric cancer [J]. Chinese Journal of Clinical Oncology. 2018, 45 (4): 209-213.
[18] Liu Yanfeng, Miao Mengyuan, Mao Weizheng. Advances in the molecular mechanism pathogenesis and targeted therapy of gastric cancer [J]. Journal of Preventive Medicine of Chinese People's Liberation Army. 201937 (4): 189-190. 